\title{
Inhibition of angiotensin II-induced contraction of human airway smooth muscle cells by angiotensin-(1-7) via downregulation of the RhoA/ROCK2 signaling pathway
}

\author{
NING LI ${ }^{1}$, RUIJUN CAI ${ }^{2}$, YI NIU ${ }^{1}$, BIN SHEN $^{1}$, JIAN XU $^{3}$ and YUANXIONG CHENG ${ }^{1}$ \\ Departments of ${ }^{1}$ Respiratory Diseases, ${ }^{2}$ Thoracic Cardiovascular Surgery and ${ }^{3}$ Kidney Transplantation, \\ Nanfang Hospital, Southern Medical University, Guangzhou, Guangdong 510515, P.R. China
}

Received May 4, 2012; Accepted July 3, 2012

DOI: $10.3892 / \mathrm{ijmm} .2012 .1080$

\begin{abstract}
Sustained renin-angiotensin system (RAS) activation in asthmatic patients plays a crucial role in airway hyperresponsiveness and airflow limitation. Angiotensin II (Ang II), as a key peptide of RAS, contributes to the contraction of human airway smooth muscle by activating the RhoA/ Rho-associated coiled-coil containing protein kinase 2 (ROCK2) signaling pathway. Angiotensin-(1-7) [Ang-(1-7)], is a component of the angiotensin I converting enzyme 2 (ACE2)-Ang-(1-7)-Mas axis which counteracts the detrimental effects of the ACE- Ang II-angiotensin type 1 receptor (AT1R) axis in vivo; however, whether Ang-(1-7) can inhibit the effect of Ang II in the contraction of human airway smooth muscle cells (HASMCs) is unknown. In our study, collagen gel lattices and immunofluorescence were used to evaluate the contraction of HASMCs induced by Ang II. Real-time PCR and western blot analysis were performed to confirm the regulatory mechanism and the participating signaling pathway. Ang II caused the contraction of HASMCs; this effect was reversed by Ang-(1-7). In addition, irbesartan and A779, which are inhibitors of AT1R and Mas, respectively, attenuated the effect of Ang II and Ang-(1-7). Furthermore, Y-27632, an inhibitor of ROCK2, attenuated the Ang II-induced contraction of HASMCs by blocking the RhoA/ROCK2 signaling pathway which is involved in this contraction, and thus may be a major regulator involved in the basal maintenance of contractility in HASMCs. These data demonstrate that Ang II induces the contraction of HASMCs and that this effect can be reversed by Ang-(1-7), partially through the downregulation of of the RhoA/ROCK2 signaling pathway.
\end{abstract}

Correspondence to: Dr Yuanxiong Cheng, Department of Respiratory Diseases, Nanfang Hospital, Southern Medical University, No. 1838 Guangzhou Avenue North, Guangzhou, Guangdong 510515, P.R. China

E-mail: drchengyx@yahoo.com.cn

Key words: human airway smooth muscle cell contraction, angiotensin II, angiotensin-(1-7), RhoA/Rho-associated coiled-coil containing protein kinase 2 signaling pathway

\section{Introduction}

Airway narrowing is the final common pathway leading to symptoms and physiological changes in asthma. Airway smooth muscle contraction in response to multiple bronchoconstrictor mediators and neurotransmitters is the predominant mechanism of airway narrowing. The lung has the capacity to locally generate angiotensin II (Ang II) $(1,2)$. Under the pathological conditions of asthma, with antigen sensitization and challenge, the airway renin-angiotensin system (RAS) is triggered, thereby elevating the Ang II concentration, which then contributes to bronchoconstriction and bronchial hyperactivity $(3,4)$. New constituents of RAS, angiotensin-(1-7) [Ang-(1-7)] and its main inducer, angiotensin I converting enzyme 2 (ACE2), have been discovered. As opposed to Ang II, Ang-(1-7) has been shown to suppress heart or vascular muscle remodeling, proliferation, migration, inflammation and fibrosis (5-7). Ang-(1-7) acting via its receptor, Mas (8,9), is considered a protective factor, with beneficial effects as opposed to the Ang II-angiotensin type 1 receptor (AT1R), particularly in acute lung injury induced by severe acute respiratory syndrome (SARS) $(10,11)$. As previously reported, AT1R expression increased in patients with asthma associated with airway remodeling and dysfunction; however, valsartan, an AT1R antagonist, inhibits AT1R expression and partially inhibits structural airway changes in chronic ovalbuminexposed rats (12).

Ang II activates the small guanosine triphos-phatase (GTPase), RhoA, and its downstream effector, Rho-associated coiled-coil containing protein kinase 2 (ROCK2), which plays a main role in the intensity and persistence of vascular smooth muscle cell contraction and vasoconstriction $(13,14)$. According to recent research, Ang II acts via AT1R in vascular smooth muscle cells $(15,16)$, hepatic stellate cells (17) and airway smooth muscle cells $(18,19)$. The RhoA/ROCK2 signaling pathway also plays a crucial role in biological functions, including contraction, migration and immunoregulation. Rho kinase activation is associated with the maintance of the contractive phenotype of human airway smooth muscle cells (HASMCs) (18).

In the present study, we investigated the possible effects of Ang-(1-7) on HASMC contraction. We hypothesized that 
Ang-(1-7) may inhibit Ang II-induced airway smooth muscle cell contraction via the Mas receptor. We further hypothesized that Ang-(1-7) treatment may suppress the Ang II-induced activation of the RhoA/ROCK signaling pathway, a possible mechanism for its inhibitory effect on smooth muscle cell contraction.

\section{Materials and methods}

Ethics statement. All experimental procedures and protocols in this study were approved by the Ethics Committee of Nanfang Hospital, Southern Medical University (NFEC201109-K1). Prior to the experiments, the patients were informed of the objectives and provided written informed consent to participate in the study.

Cell isolation and culture. HASMCs were isolated from the lobar or main bronchus obtained from lung resection donors, approved by the Division of Thoracic Surgery. The cells were maintained as primary culture in Dulbecco's modified Eagle's medium (DMEM; Hyclone) with $10 \%$ fetal bovine serum (FBS; Hyclone), $100 \mathrm{U} / \mathrm{ml}$ penicillin and $100 \mathrm{U} / \mathrm{ml}$ streptomycin. The morphology and phenotype of the cells was identified, with a purity of $\geq 95 \%$. Cells from passages $3-8$ were grown to confluence and were harvested by trypsin digestion and used for the experiments. To evaluate whether Ang II affects HASMCs in a time-dependent manner, Ang II was used to stimulate the cells for $5,15,30$ or $60 \mathrm{~min}$, at a concentration of $10^{-7} \mathrm{M}$ under serum-free conditions. In all other experiments, $10^{-7} \mathrm{~mol} / 1$ Ang II with or without Ang-(1-7) was added to the cell cultures followed by incubation for 15 min. Y-27632 (a ROCK-2 inhibitor) and irbesartan (IRB, an AT1R inhibitor) were used at a concentration of $10^{-5} \mathrm{M}$, $0.5 \mathrm{~h}$ prior to the addition of Ang II $\left(10^{-7} \mathrm{M}\right)$.

Gel contraction assay. HASMCs were treated with IRB $\left(10^{-5} \mathrm{M}\right)$ or Y-27632 $\left(10^{-5} \mathrm{M}\right)$ for $30 \mathrm{~min}$, then Ang II $\left(10^{-7} \mathrm{M}\right)$ with or without Ang-(1-7) $\left(10^{-7} \mathrm{M}\right)$ was added to the culture medium and the cells were then added to the collagen suspension. Buffer without Ang II was used as the control. Type 1 rat-tail collagen suspension $(5 \mathrm{mg} / \mathrm{ml}$; Shengyou Biotechnology Co., Ltd.) was prepared according to the manufacturer's instructions. The final collagen suspension $(1 \mathrm{mg} / \mathrm{ml})$ containing the HASMCs in $0.76 \mathrm{ml}\left(1 \times 10^{5}\right.$ cells) with or without pre-treatment, was cast in $35-\mathrm{mm}$ culture plates and allowed to polymerize $\left(20 \mathrm{~min}, 37^{\circ} \mathrm{C}\right)$. Once polymerized, the gels were carefully detached from the culture plates and filled with serum-free medium. The gels were equilibrated overnight after detachment to avoid the initial contraction. The surface area of the collagen gels was measured using Image J analysis software. All experiments were performed in triplicate. The relative maximum was expressed by the formula: [(gel surface area of control - gel surface area of test substance)/gel surface area of control] $\times 100 \%$.

Immunofluorescence. HASMCs were cultured on slides at a density of $5 \times 10^{4}$ cells $/ \mathrm{cm}^{2}$ and incubated for $24 \mathrm{~h}$ to permit cell attachment. Under serum-free conditions, the cells were treated with various reagents as described in the section 'Cell isolation and culture'. Subsequently, the cells were fixed with $3.7 \%$ paraformaldehyde for $10 \mathrm{~min}$. After washing with phosphate-buffered saline (PBS), the cells were treated with $0.1 \%$ Triton $\mathrm{X}-100$ for 5 min to permeabilize them. To visualize F-actin stress fibers, the cells were stained with $2 \mu \mathrm{g} / \mathrm{ml}$ of TRITC-conjugated phalloidin (Sigma) for $20 \mathrm{~min}$ at room temperature and then restained with 4'-6-diamidino2-phenylindole (DAPI, BioTime) for the visualization of the nuclei. After mounting, images were obtained using an inverted fluorescence microscope (Olympus-FL 500; Tokyo, Japan).

Real-time PCR. Total RNA was isolated from the HASMCs by using the RNAiso Plus kit. The cDNA was generated by the PrimeScript ${ }^{\circledR}$ RT reagent kit (from Takara Biotechnology Co., Ltd.). In order to analyze messenger RNA (mRNA), we synthesized the template cDNA for the subsequent PCR analyses. Real-time PCR analysis of the rat genes was performed by the use of SYBR-Green-based assays with the ABI 7500 Real-Time PCR System (Applied Biosystems, Foster City, CA, USA), using SYBR ${ }^{\circledR}$ Premix Ex Taq ${ }^{\mathrm{TM}}$ II (from Takara Biotechnology Co., Ltd.). The human RhoGEF (NM-198997), RhoAGTP (NM-001664.2) and ROCK2 (NM-021804.2) primers were designed by Primer 5.0 software (Table I). The primers were used at a concentration of $0.4 \mu \mathrm{M}$ in each reaction. Cycling conditions were as follows: step 1, $30 \mathrm{sec}$ at $95^{\circ} \mathrm{C}$; step $2,5 \mathrm{sec}$ at $95^{\circ} \mathrm{C}$ and $34 \mathrm{sec}$ at $60^{\circ} \mathrm{C}$; step $3,15 \mathrm{sec}$ at $95^{\circ} \mathrm{C}, 1 \mathrm{~min}$ at $60^{\circ} \mathrm{C}$ and $15 \mathrm{sec}$ at $95^{\circ} \mathrm{C}$, with repetition of step 2 for 35 times. Data from the reaction were collected and analyzed by the 7500 software v2.0.4, using a standard curve. The relative quantification of gene expression was normalized to GAPDH.

Western blot analysis. Total protein was extracted using the radioimmunoprecipitation assay (RIPA) lysis buffer (Beyotime Biotech, Haimen, China), containing protease inhibitors (Roche, Basel, Switzerland) or phosphatase inhibitors (Sigma). Protein samples $(20 \mu \mathrm{g})$ were heated at $100^{\circ} \mathrm{C}$ for $7 \mathrm{~min}$ before loading and separation on 10-12\% sodium dodecyl sulfate polyacrylamide gel electrophoresis (SDS-PAGE). Proteins were transferred onto a polyvinylidene fluoride (PVDF) membranes (Bio-Rad, Hercules, CA, USA). Subsequently, the membranes were blocked with $5 \%$ bovine serum albumin in TBST buffer (20 mM Tris, $500 \mathrm{mM} \mathrm{NaCl}$ and $0.1 \%$ Tween-20) for $1 \mathrm{~h}$ at room temperature. The membranes were then incubated at $4^{\circ} \mathrm{C}$ overnight with various primary antibodies at a 1:1,000 dilution in $5 \%$ bovine serum albumin buffer. The following primary antibodies were used: rabbit anti-phosphorylated myosin light chain (P-MLC), rabbit anti-phospho-moesin, rabbit anti-MLC and rabbit anti-moesin (all from Cell Signaling Technology, Danvers, MA, USA). To visualize the positive protein bands, horseradish peroxidase-linked secondary antibodies and the ECL system (Thermo, USA) were used. The density of the individual bands developed on radiographic film was then quantified using a densitometric scanner with Gel-Pro Analyzer 4 software.

Statistical analyses. All data are presented as the means \pm SEM, based on experiments repeated in triplicate, unless otherwise specified. Multiple comparisons were analyzed using one-way analysis of variance (ANOVA) with the Statistical Package for 
Table I. Primers used in the real-time PCR analyses.

\begin{tabular}{lll}
\hline Gene name & Accession no. & \multicolumn{1}{c}{ Primers } \\
\hline ARHGEF1 & NM_198997.1 & 5'-AACCAAGCCGTGCGTGAC-3' \\
& & 5'-TGAACTCGCTCAGCATAGGG-3' \\
RhoAGTP & NM_001664.2 & 5'-AGATATGGCAAACAGGATT-3' \\
& & 5'-TTTCACAAGACAAGGCAC-3' \\
ROCK2 & NM_021804.2 & 5'-TATGCGGATTCACTTGTAG-3' \\
& & 5'-AGTCGTACCTCCCTATCTG-3' \\
\hline
\end{tabular}
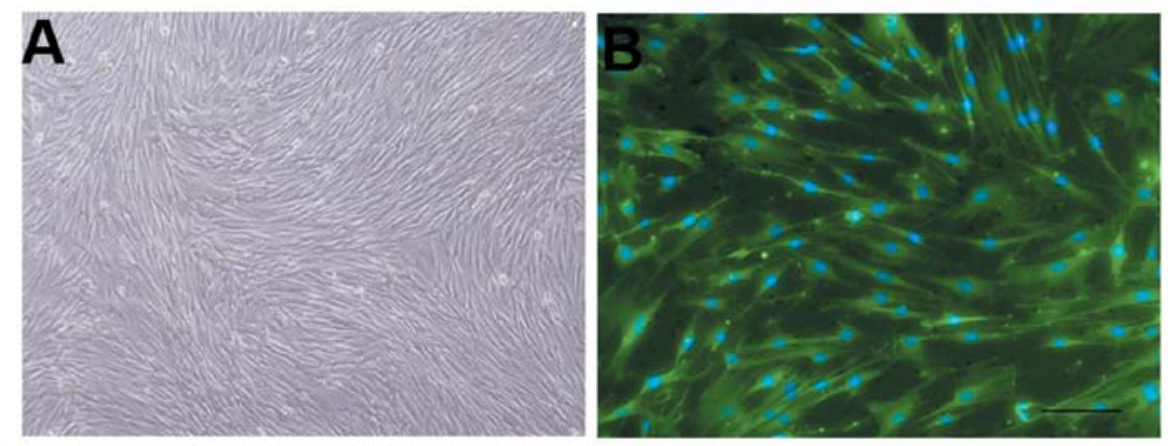

Figure 1. Primarily cultured HASMCs were identified by their typical morphology and phenotype. (A) The confluent cells displayed the typical 'hill and valley' appearance. (B) HASMCs immunostained with anti- $\alpha$-actin antibody revealed the positive expression of $\alpha$-actin. (scale bar, $200 \mu \mathrm{m}$ ).

Social Sciences (SPSS) 13.0 (Statistical Software for Social Sciences, Chicago, IL). Differences were considered to be statistically significant when $\mathrm{P}<0.05$.

\section{Results}

Cell culture. Primary cultured HASMCs displayed the typical 'hill and valley' appearance when confluenced under an inverted light microscope. HASMCs were initially characterized by positive immunostaining for $\alpha$-smooth muscle actin ( $\alpha$-SMA). There they were identified by their typical morphology and phenotype, degree of purity $\geq 95 \%$ (Fig. 1).

Ang II induction of collagen gel contraction. In order to validate whether Ang II induces the contraction of HASMCs embedded in collagen gel, a hydrated collagen gel assay was utilized. Fig. 2 displays a representative experiment with collagen lattice contraction. As shown in Fig. 2 (upper panel), the control group exposed to gel buffer only, demonstrated a minimal decrease in the collagen surface area. Compared with the control cells, Ang II at $10^{-7} \mathrm{M}$ caused an obvious decrease in surface gel area. The cells co-treated with Ang II and Ang-(1-7) demonstrated a slight decrease in the collagen surface area compared with Ang II, and A779 reversed the effect of Ang-(1-7) by blocking the Mas receptor. The addition of the inhibitors, Y-27632 and IRB, to the HASMCs for $0.5 \mathrm{~h}$ prior to a 60-min exposure to Ang II, resulted in significant loss of gel contraction compared with Ang II alone (Fig. 2, upper panel). The quantification of percentage gel contraction is shown in Fig. 2 (bottom panel).
Ang II induction of increased actin stress fiber formation. Primary cultured HASMCs developed a fusiform shape, with some quantity and a regular arrangement of actin stress fibers. The Fig. 3 showed cytoskeletal reorganizations triggered by Ang II $\left(10^{-7} \mathrm{M}, 60 \mathrm{~min}\right)$ compared with the other groups in sparsely cultivated HASMCs typically stained with fluorophore-conjugated phalloidin with increased mass and stress fibers. Within $30 \mathrm{~min}$ of co-treatment with Ang II and Ang-(1-7), staining of the F-actin stress fibers showed a significant decrease in stress fibers in the HASMCs, compared with treatment with Ang II alone. A779 blocked the effect of Ang-(1-7), and the Ang II-induced increase in actin stress fiber formation was partially reversed by treatment with Y-27632 or IRB (Fig. 3).

Ang II activates the RhoA/ROCK-2 signaling pathway. Ang II induces various pathological effects through AT1R, such as contraction, migration, cell growth or hypertrophy, via different signaling pathways (20). However, the mechanisms behind the effect of Ang II on HASMCs and the role of the RhoA/ROCK2 signaling pathway remain unknown. Therefore, we performed real-time PCR in ordr to evaluate the expression of the genes, ARHGEF1, RhoAGTP and ROCK2, which are key molecules of the RhoA/ROCK2 signaling pathway. Following treatment with Ang II, the expression of ARHGEF1, RhoAGTP and ROCK2 in the HASMCs increased, indicating the activation of the RhoA/ROCK2 pathway (Fig. 4). Ang-(1-7) partially reversed the increase in ARHGEF1, RhoAGTP and ROCK2 expression, similar to IRB and Y-37632. 

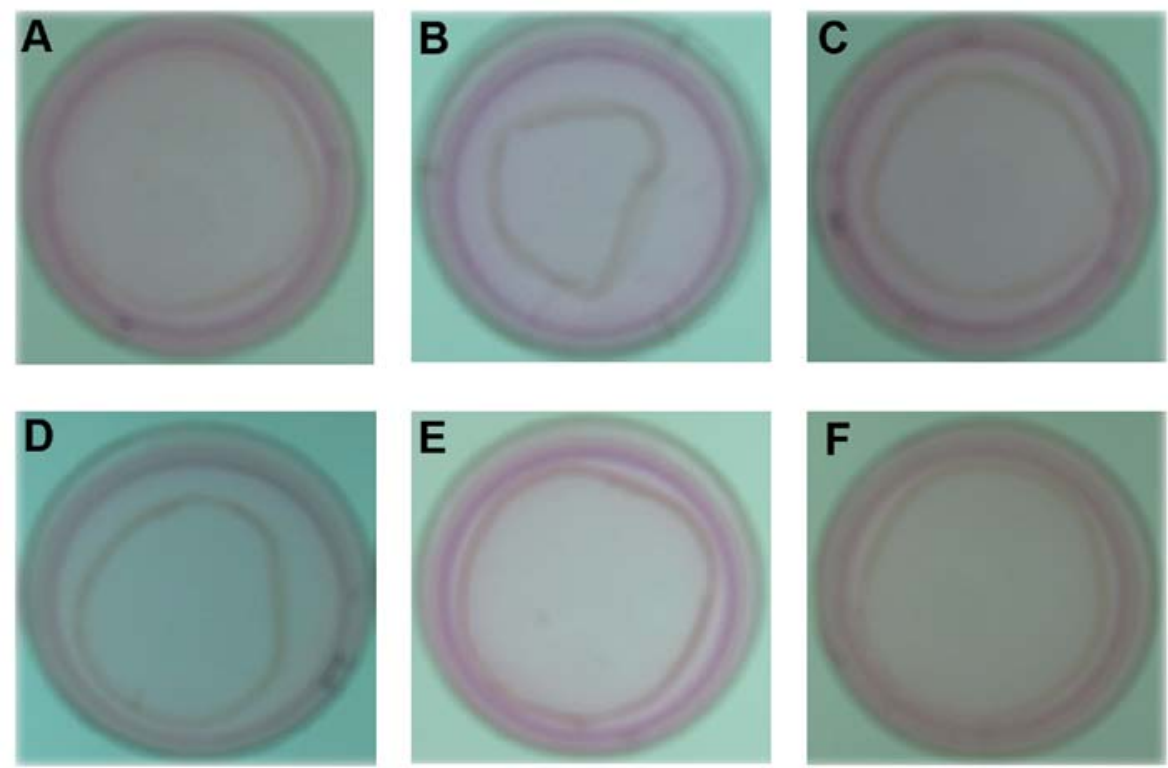

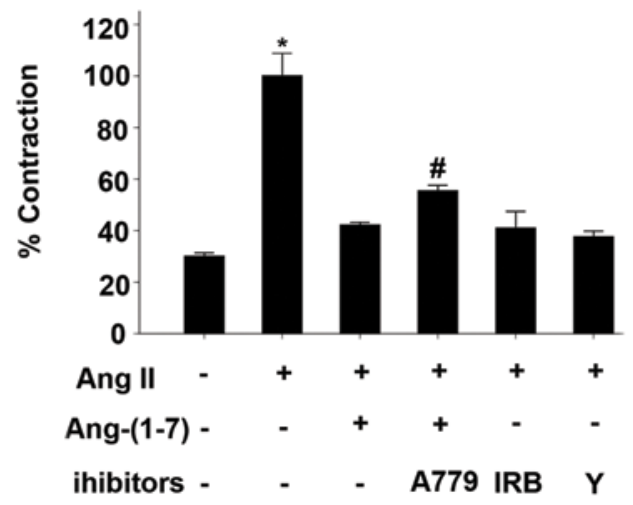

Figure 2. Mean maximal contraction of collagen gels with various stimuli, compared with the initial surface area. Upper panel: representative images of gel contraction in response to various stimuli. Lower panel: After incubation with buffer, the cells were treated with $10^{-7} \mathrm{M}$ Ang II and Ang-(1-7) with or without pre-treatment with $10^{-5} \mathrm{M}$ A779, $10^{-5} \mathrm{M}$ irbesartan (IRB) or $10^{-5} \mathrm{M}$ Y-27632 (Y, a ROCK-2 inhibitor) followed treatment with $10^{-7} \mathrm{M}$ Ang II for 60 min. A, Control; B, Ang II; C, Ang II + Ang-(1-7); D, Ang II + Ang-(1-7) + A779; E, Ang II + IRB; F, Ang II + Y-27632 ("P<0.05 vs. the other groups; ${ }^{\text {"}} \mathrm{P}<0.05$ vs. the other groups).
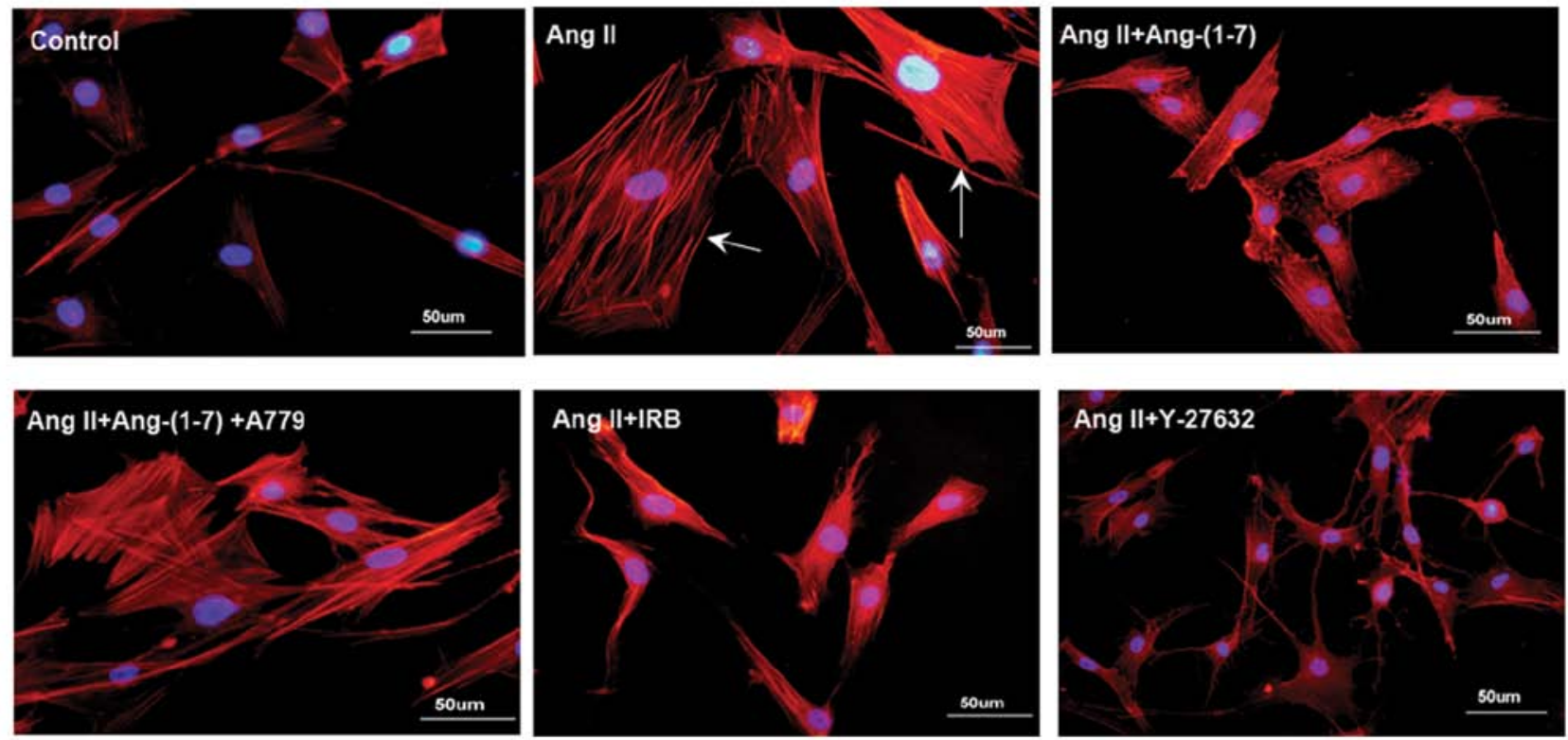

Figure 3. Cytoskeletal reorganizations triggered by the Ang II treatment $\left(10^{-7} \mathrm{M}, 60 \mathrm{~min}\right)$ of HASMCs. The staining HASMCs with TRITC-phalloidin showed cytoskeletal changes in the corresponding group F-actin (red) and DAPI (blue). After a 60-min exposure to $10^{-7} \mathrm{M}$ Ang II, the cells had increased numbers of stress fibers and developed fiber-positive projections. Following costimulation with Ang II and Ang-(1-7), there were fewer stress fibers. Furthermore, the cells were pre-incubated for 30 min with A779, an inhibitor of Mas, irbesartan (IRB), or Y-27632 (Y) at a concentration of $10^{-5} \mathrm{M}$ and then treated with Ang II or co-treated with Ang II and Ang-(1-7) for $60 \mathrm{~min}$. The Ang II-induced increase in stress fiber formation was partially inhibited by Ang-(1-7), IRB and Y-27632 (Y). In addition, the effect of Ang-(1-7) was partially reversed by A779. All scale bars, $50 \mu \mathrm{m}$. 

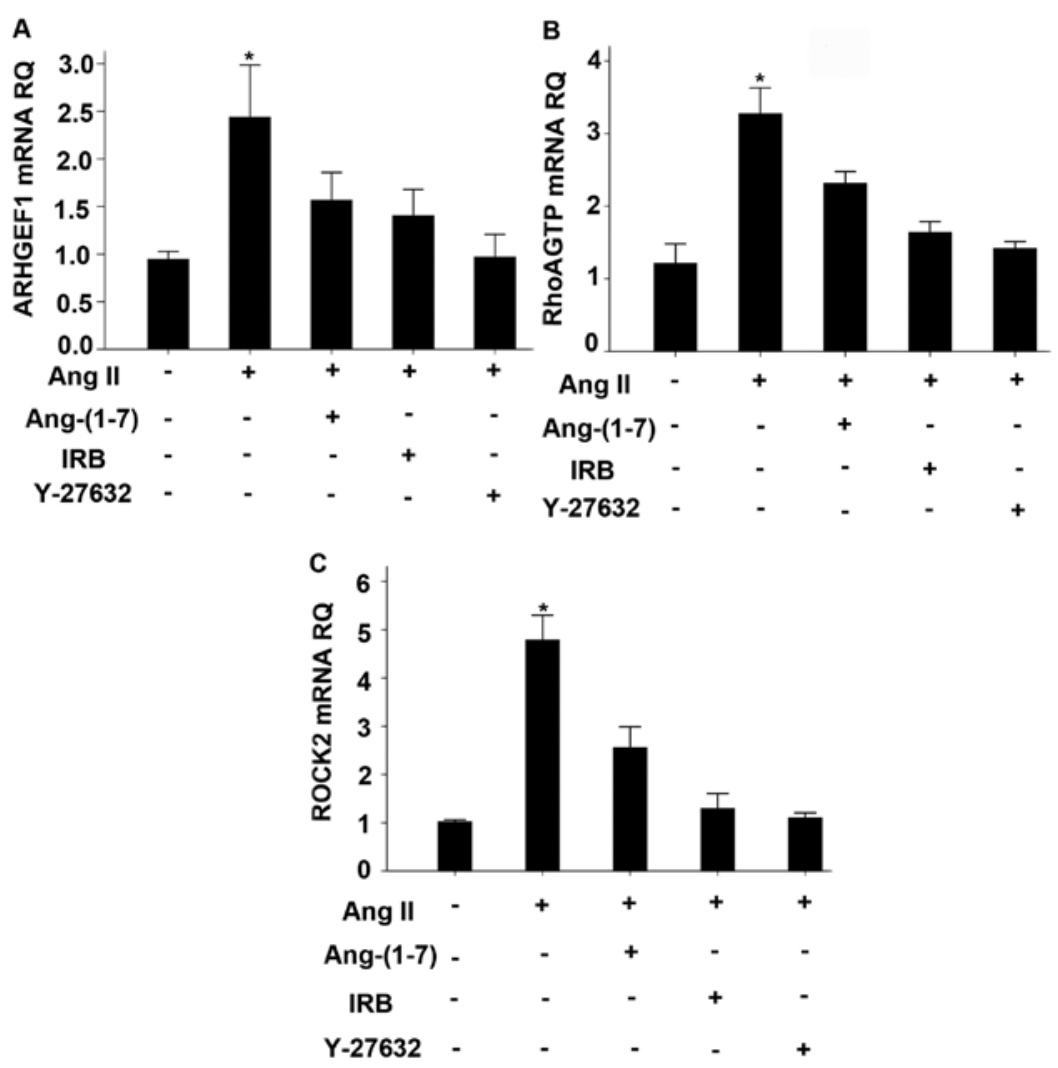

Figure 4. The RhoA/ROCK2 signaling pathway participates in the HASMC contraction induced by Ang II. Following exposure to $10^{-7} \mathrm{M}$ Ang II for $30 \mathrm{~min}$, the relative amounts of the genes, (A) ARHGEF1, (B) RhoAGTP and (C) ROCK2 increased significantly. After costimulation with Ang II and Ang-(1-7), or pre-incubation with irebesartan (IRB) and Y-27632 (Y), the relative gene expression (RQ) was attenuated significantly ("P $<0.05$ vs. the other groups).

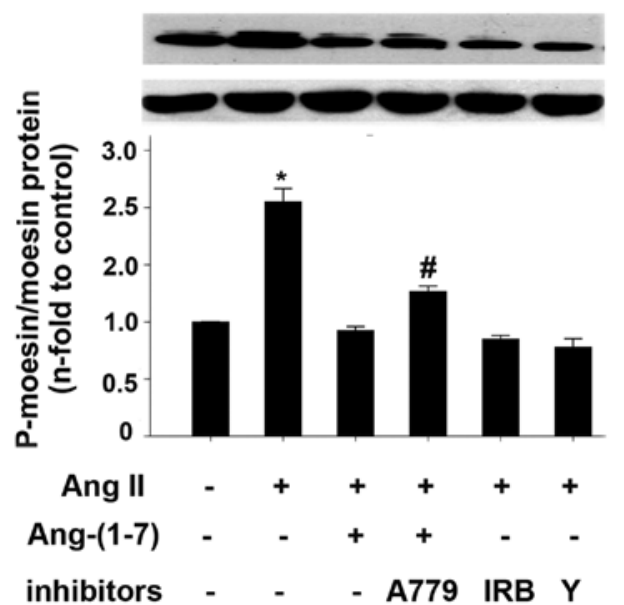

Figure 5. Representative immunoblotting and quantitative analysis of P-moesin and moesin protein expression in HASMCs. HASMCs were stimulated with Ang II with or without Ang-(1-7) and the inhibitors (Ang II and Ang-(1-7), $10^{-7} \mathrm{M}$, for $30 \mathrm{~min}$ ); HASMCs were pre-treated with A779, irbesartan (IRB) and $\mathrm{Y}-27632(\mathrm{Y})$, for $30 \mathrm{~min}$ at a concentration of $10^{-5} \mathrm{M}\left({ }^{*} \mathrm{P}<0.05\right.$ vs. the other groups; ${ }^{"} \mathrm{P}<0.05$ vs. the other groups).

Ang II enhances the level of phosphorylated moesin (P-moesin) and $P-M L C$. In order to elucidate the mechanism by which Ang II induces HASMC contraction, we then determined how the Ang II-treated HASMCs respond to Ang-(1-7), IRB and Y-27632 co-treatment compared to the untreated HASMCs. Once the RhoA/ROCK pathway was activated, increasing
RhoAGTP levels activated the ROCK2 synthesis, which was reflected by the phosphorylation of moesin, as shown by western blot analysis in Fig. 5. Furthermore, moesin [a member of the ezrin/radixin/moesin (ERM) family of proteins], a substrate of ROCK2, provides a crucial link with the F-actin cytoskeleton and membrane proteins at the cell periphery (21). The P-moesin protein was assayed to elucidate how ERM proteins affect the cell cytoskeleton and contraction function.

Furthermore, to evaluate the effect of Ang II on MLC expression, we exposed the HASMCs to Ang II for different amounts of time, as described in Materials and methods, then measured P-MLC protein expression by western blot analyses. Fig. 6 shows the optical time of the Ang II stimulation of HASMCs, which is in accordance with a previous study (22). The Ang II stimulatin of HASMCs occurred in a time-dependent manner. As shown, Ang II induced an increase in P-MLC expression as early as $5 \mathrm{~min}$ and this increase peaked at $15 \mathrm{~min}$.

One of the major consequences of the activation of the RhoA/ROCK2 pathway is the phosphorylation of MLC, which is critically involved in the contraction of HASMCs. As demonstrated in Fig. 7, the exposure of the activated HASMCs to Ang II led to increased amounts of P-MLC. These effects were significantly inhibited by co-treatment with Ang-(1-7), IRB and Y-27632. Similarly, pre-treatment with A779 attenuated the inhibitory effect of Ang-(1-7) in response to Ang II stimulation. The above results suggest that Ang-(1-7) negatively regulates the contractile response of HASMCs to Ang II via the inhibition of the RhoA/ROCK2 pathway. 


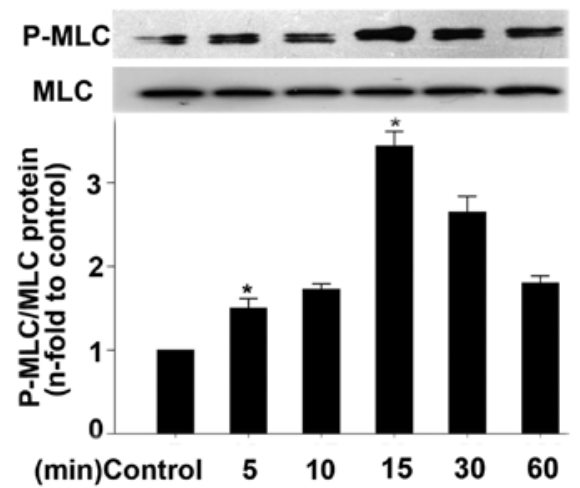

Figure 6. Time-dependence of Ang II-triggered P-MLC expression. HASMCs were treated with $10^{-7} \mathrm{M}$ Ang II for different periods of time and then lysed. P-MLC and MLC expression was assessed by western blot analysis and quantified as described in Materials and methods.

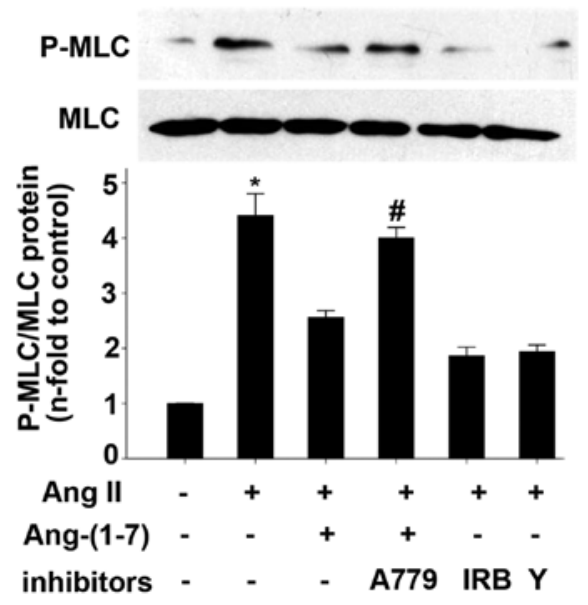

Figure 7. P-MLC/MLC expression in HASMCs treated with various stimuli. Ang II $\left(10^{-7} \mathrm{M}\right)$, with or without Ang-(1-7), was added to the cell cultures and the cells were incubated for $30 \mathrm{~min}$. Y-27632 (Y), irbesartan (IRB) and A779 were used at a concentration of $10^{-5} \mathrm{M}, 0.5 \mathrm{~h}$ prior to the addition of Ang II $\left(10^{-7} \mathrm{M}\right) .{ }^{*} \mathrm{P}<0.05$ vs. the other groups; ${ }^{*} \mathrm{P}<0.05$ vs. the other groups.

\section{Discussion}

The results from the present study demonstrated that Ang II $\left(10^{-7} \mathrm{M}\right)$ caused the significant contraction of primary cultured HASMCs in embedded in collagen gels. This effect was inhibited by IRB and Y-27632. Ang-(1-7) suppressed the contraction of HASMCs caused by Ang II and the inhibitory effects of Ang-(1-7) were partially reversed by the Ang-(1-7) receptor antagonist, A799. The difference in contraction among the groups was confirmed by immunofluorescence. Ang II, a key peptide fragment of RAS, was originally described as an important regulator of blood pressure and electrolytic balance, while Ang-(1-7) has demonstrated cardiovascular protection. Ang II is considered to induce the contraction of airway smooth muscle and bronchoconstriction directly and potentiates methacholine (Mch)-induced bronchoconstriction in mild asthmatic patients (23). A previous meta-analysis suggested that the insertion/deletion (I/D) polymorphism of the ACE gene, a key gene in Ang II synthesis, is a risk factor for asthma (24). In the current study, we demonstrate the role of
Ang II in HASMC contraction and investigated the potential mechanism involved.

Collagen gel contraction assay is a common method used to examine several constrictors, such as cysteinyl leukotrienes (cysLTD4), and has been used to measure Mch-induced contractile capacity in established human bronchial smooth muscle cells $(25,26)$. It had been used to examine the contractile capacity of HASMCs between asthmatic and non-asthmatic patients (27). In our study, we found out that Ang II as a vasoconstrictor, induced an increase in the contractility of HASMCs. LTD4 and Mch had a similar effect.

Ang II and Ang-(1-7) are considered as the devil and angel' in RAS (6), one counteracting the effects of the other. Ang-(1-7) plays a protective role through the Mas receptor by inhibiting AT1R. However, it is not clear which intracellular signaling pathway is invovled. Several factors, such as extracellular signal-regulated kinase (ERK) 1/2 phosphorylation, ERK Akt phosphorylation and mitogen-activated protein (MAP) kinase inhibition seem to be involved in this signaling pathway (28-30). A previous study indicated that the chronic hypotensive effects of losartan in normal rats were mediated in part through the action of Ang-(1-7) (31). Another study showed that the Mas receptor is essential in mediating the endothelium-dependent relaxation response induced by perivascular adipose tissue, thus highlighting the important role of Ang-(1-7) (32). In the lung, Ang-(1-7) inhibits the apoptosis of alveolar epithelial cells (AECs) through Mas and ACE-2 regulates the AEC survival by balancing pro-apoptotic Ang II and its anti-apoptotic degradation product, Ang-(1-7) (33). Further studies are required to clarify the roles of Ang-(1-7) in vasodilatation, natriuresis, anti-proliferation and the increase in the bradykinin-nitric oxide (NO) system, counteracting the effects of Ang II. Therefore, we investigated whether Ang-(1-7) has an inhibitory effect on the Ang II-induced contraction of HASMCs.

In our study, the receptor inhibitors, IRB and Mas, were used to elucidate the potential mechanism of cell contraction, stress fiber formation and corresponding protein changes. Our results confirmed that the contraction of HASMCs in response to Ang II is mediated through the well-characterized RhoA/ROCK2 signal pathway.

As previously reported, in vascular cells, endogenous ACE2 counteracts the Ang II-mediated cellular response partly by the upregulation of Ang-(1-7) through Mas (30). However, in our study, we proved that Ang-(1-7) inhibits Ang II-mediated cellular contraction via the downregulation of the RhoA/ ROCK2 signaling pathway. ROCK2, a cytosolic small GTPase Rho kinase, plays a predominant role in vascular smooth muscle cell contractility (14). The activation of RAS also leads to the phosphorylation of moesin downstream of Rho and MLC. Moreover, previous studies have proven that Ang II activates the RhoA/ROCK-2 signaling pathway in vascular smooth muscle cells $(34,35)$.

However, to date, no information is available on the mechanisms causing the contraction induced by Ang II in HASMCs at the cellular level. In our study, the inhibition of the RhoA/ ROCK 2 signaling pathway was clearly demonstrated by the inhibition of the contraction of HASMCs in the group pre-treated with Y-27632, as the phosphorylation of MLC downstream of Rho was decreased significantly. 
From the above results, we hypothesized that Ang-(1-7) may weaken the effects of Ang II by downregulating the RhoA/ROCK2 signaling pathway. The activation of key molecules, including RhoGEFs, RhoAGTP and ROCK2, was associated with the contraction of HASMCs and cytoskeletal rearrangement. Y-27632, as a ROCK2 inhibitor, demonstrated a significant inhibitory effect on the increased contraction by inhibiting the increase in stress fibers. This correlated with the dephosphorylation of moesin. Traction forces generated by the increase in stress fibers induced by the addition of Ang II leads to a significant increase in cell contractility $(36,37)$.

In conclusion, our study reveals a function of RAS in regulating airway narrowing through its induction of intense HASMC contraction. Further studies are required to better understand the underlying mechanism; however, the RhoA/ ROCK2 signaling pathway plays a key role in the Ang II/ Ang-(1-7)-mediated regulation of HASMC contraction.

\section{Acknowledgements}

This study was supported by the Presidential Foundation of Nanfang hospital (grant no. 2009B007). We would like to thank the Department of Thoracic Surgery of our hospital for its valuable contribution of the lung specimens.

\section{References}

1. Phillips MI, Speakman EA and Kimura B: Levels of angiotensin and molecular biology of the tissue renin angiotensin systems. Regul Pept 43: 1-20, 1993.

2. Marshall RP, Gohlke P, Chambers RC, Howell DC, Bottoms SE, Unger T, McAnulty RJ and Laurent GJ: Angiotensin II and the fibroproliferative response to acute lung injury. Am J Physiol Lung Cell Mol Physiol 286: L156-L164, 2004.

3. Cojocaru E, Dumitriu IL, Bogdan G, Costuleanu M, Slatineanu SM and Petrescu G: Involvement of angiotensin on adenosine-induced bronchial hyperreactivity. Pneumologia 58: 19-23, 2009 (In Romanian).

4. Veerappan A, Reid AC, Estephan R, O'Connor N, ThadaniMulero M, Salazar-Rodriguez M, Levi R and Silver RB: Mast cell renin and a local renin-angiotensin system in the airway: role in bronchoconstriction. Proc Natl Acad Sci USA 105: 1315-1320, 2008.

5. Zhong J, Basu R, Guo D, Chow FL, Byrns S, Schuster M, Loibner H, Wang XH, Penninger JM, Kassiri Z and Oudit GY: Angiotensin-converting enzyme 2 suppresses pathological hypertrophy, myocardial fibrosis and cardiac dysfunction. Circulation 122: 717-728, 2010.

6. Iwai M and Horiuchi M: Devil and angel in the renin-angiotensin system: ACE-angiotensin II-AT1 receptor axis vs. ACE2angiotensin-(1-7)-Mas receptor axis. Hypertens Res 32: 533-536, 2009.

7. Grobe JL, Mecca AP, Lingis M, Shenoy V, Bolton TA, Machado JM, Speth RC, Raizada MK and Katovich MJ: Prevention of angiotensin II-induced cardiac remodeling by angiotensin-(1-7). Am J Physiol Heart Circ Physiol 292: H736-H742, 2007.

8. Esteban V, Heringer-Walther S, Sterner-Kock A, de Bruin R, van den Engel S, Wang Y, Mezzano S, Egido J, Schultheiss HP, Ruiz-Ortega $\mathrm{M}$ and Walther T: Angiotensin-(1-7) and the $\mathrm{g}$ protein-coupled receptor MAS are key players in renal inflammation. PLoS One 4: e5406, 2009.

9. Santos RA, Simoes e Silva AC, Maric C, Silva DM, Machado RP, de Buhr I, Heringer-Walther S, Pinheiro SV, Lopes MT, Bader M, et al: Angiotensin-(1-7) is an endogenous ligand for the $G$ protein-coupled receptor Mas. Proc Natl Acad Sci USA 100: 8258-8263, 2003.

10. Li W, Moore MJ, Vasilieva N, Sui J, Wong SK, Berne MA, Somasundaran M, Sullivan JL, Luzuriaga K, Greenough TC, et al: Angiotensin-converting enzyme 2 is a functional receptor for the SARS coronavirus. Nature 426: 450-454, 2003.
11. Hamming I, Timens W, Bulthuis ML, Lely AT, Navis GJ and van Goor H: Tissue distribution of ACE2 protein, the functional receptor for SARS coronavirus. A first step in understanding SARS pathogenesis. J Pathol 203: 631-637, 2004.

12. Wang T, Yin KS, Liu KY, Lu GJ, Li YH and Chen JD: Effect of valsartan on the expression of angiotensin II receptors in the lung of chronic antigen exposure rats. Chin Med J (Engl) 121: 2312-2319, 2008.

13. Kogata N, Tribe RM, Fassler R, Way M and Adams RH: Integrin-linked kinase controls vascular wall formation by negatively regulating Rho/ROCK-mediated vascular smooth muscle cell contraction. Genes Dev 23: 2278-2283, 2009.

14. Wang Y, Zheng XR, Riddick N, Bryden M, Baur W, Zhang X and Surks HK: ROCK isoform regulation of myosin phosphatase and contractility in vascular smooth muscle cells. Circ Res 104: 531-540, 2009.

15. Kimura K and Eguchi S: Angiotensin II type-1 receptor regulates RhoA and Rho-kinase/ROCK activation via multiple mechanisms. Focus on 'Angiotensin II induces RhoA activation through SHP2-dependent dephosphorylation of the RhoGAP p190A in vascular smooth muscle cells'. Am J Physiol Cell Physiol 297: C1059-C1061, 2009.

16. Ohtsu H, Mifune M, Frank GD, Saito S, Inagami T, Kim-Mitsuyama S, Takuwa Y, Sasaki T, Rothstein JD, Suzuki H, et al: Signal-crosstalk between Rho/ROCK and c-Jun $\mathrm{NH} 2$-terminal kinase mediates migration of vascular smooth muscle cells stimulated by angiotensin II. Arterioscler Thromb Vasc Biol 25: 1831-1836, 2005.

17. Li J, Kuruba R, Wilson A, Gao X, Zhang Y and Li S: Inhibition of endothelin-1-mediated contraction of hepatic stellate cells by FXR ligand. PLoS One 5: e13955, 2010.

18. Gosens R, Schaafsma D, Meurs H, Zaagsma J and Nelemans SA: Role of Rho-kinase in maintaining airway smooth muscle contractile phenotype. Eur J Pharmacol 483: 71-78, 2004.

19. LoGrasso PV and Feng Y: Rho kinase (ROCK) inhibitors and their application to inflammatory disorders. Curr Top Med Chem 9: 704-723, 2009.

20. Regitz-Zagrosek V, Neuss M, Holzmeister J, Warnecke C and Fleck E: Molecular biology of angiotensin receptors and their role in human cardiovascular disease. J Mol Med 74: 233-251, 1996.

21. Bretscher A, Edwards K and Fehon RG: ERM proteins and merlin: integrators at the cell cortex. Nat Rev Mol Cell Biol 3: 586-599, 2002.

22. Touyz RM, Yao G, Viel E, Amiri F and Schiffrin EL: Angiotensin II and endothelin-1 regulate MAP kinases through different redox-dependent mechanisms in human vascular smooth muscle cells. J Hypertens 22: 1141-1149, 2004.

23. Millar EA, Nally JE and Thomson NC: Angiotensin II potentiates methacholine-induced bronchoconstriction in human airway both in vitro and in vivo. Eur Respir J 8: 1838-1841, 1995.

24. Zhang YG, Li XB, Zhang J, Huang J, He C, Tian C, Deng Y, Wan H, Shrestha D, Yang YY and Fan H: The I/D polymorphism of angiotensin-converting enzyme gene and asthma risk: a meta-analysis. Allergy 66: 197-205, 2011.

25. Kitamura N, Kaminuma O, Kobayashi N and Mori A: A contraction assay system using established human bronchial smooth muscle cells. Int Arch Allergy Immunol 146 (Suppl 1): S36-S39, 2008.

26. Kitamura N, Kaminuma O, Ohtomo T, Kiyokawa N, Kobayashi N, Suko M and Mori A: Evaluation of cysteinyl leukotriene-induced contraction of human cultured bronchial smooth muscle cells. Int Arch Allergy Immunol 149 (Suppl 1): 83-86, 2009.

27. Matsumoto H, Moir LM, Oliver BG, Burgess JK, Roth M, Black JL and McParland BE: Comparison of gel contraction mediated by airway smooth muscle cells from patients with and without asthma. Thorax 62: 848-854, 2007.

28. Giani JF, Gironacci MM, Munoz MC, Pena C, Turyn D and Dominici FP: Angiotensin-(1 7) stimulates the phosphorylation of JAK2, IRS-1 and Akt in rat heart in vivo: role of the AT1 and Mas receptors. Am J Physiol Heart Circ Physiol 293: H1154-H1163, 2007.

29. Su Z, Zimpelmann J and Burns KD: Angiotensin-(1-7) inhibits angiotensin II-stimulated phosphorylation of MAP kinases in proximal tubular cells. Kidney Int 69: 2212-2218, 2006.

30. Hayashi N, Yamamoto K, Ohishi M, Tatara Y, Takeya Y, Shiota A, Oguro R, Iwamoto Y, Takeda M and Rakugi H: The counterregulating role of ACE2 and ACE2-mediated angiotensin 1-7 signaling against angiotensin II stimulation in vascular cells. Hypertens Res 33: 1182-1185, 2010. 
31. Collister JP and Hendel MD: The role of Ang (1-7) in mediating the chronic hypotensive effects of losartan in normal rats. J Renin Angiotensin Aldosterone Syst 4: 176-179, 2003.

32. Lee RM, Bader M, Alenina N, Santos RA, Gao YJ and Lu C: Mas receptors in modulating relaxation induced by perivascular adipose tissue. Life Sci 89: 467-472, 2011.

33. Uhal BD, Li X, Xue A, Gao X and Abdul-Hafez A: Regulation of alveolar epithelial cell survival by the ACE-2/angiotensin 1-7/ Mas axis. Am J Physiol Lung Cell Mol Physiol 301: L269-L274, 2011.

34. Cario-Toumaniantz C, Ferland-McCollough D, Chadeuf G, Toumaniantz G, Rodriguez M, Galizzi JP, Lockhart B, Bril A, Scalbert E, Loirand G and Pacaud P: RhoA guanine exchange factor expression profile in arteries: Evidence for a Rho kinasedependent negative feedback in angiotensin II-dependent hypertension. Am J Physiol Cell Physiol 302: C1394-C1404, 2012
35. Savoia C, Tabet F, Yao G, Schiffrin EL and Touyz RM: Negative regulation of RhoA/Rho kinase by angiotensin II type 2 receptor in vascular smooth muscle cells: role in angiotensin II-induced vasodilation in stroke-prone spontaneously hypertensive rats. J Hypertens 23: 1037-1045, 2005.

36. Gildner CD, Lerner AL and Hocking DC: Fibronectin matrix polymerization increases tensile strength of model tissue. Am J Physiol Heart Circ Physiol 287: H46-H53, 2004.

37. Hocking DC, Sottile J and Langenbach KJ: Stimulation of integrin-mediated cell contractility by fibronectin polymerization. J Biol Chem 275: 10673-10682, 2000. 